\title{
LE POIDS DES MOTS ET DES IMAGES DANS LES TEXTES DE PROMOTION TOURISTIQUE
}

\author{
Montserrat PLANELLES IVÁÑEZ \\ Université d'Alicante
}

\begin{abstract}
The aim of this paper is to describe the lexical and discursive aspect of advertising messages of health tourism and sports. The approach will be pragmatic, semiotic and extralinguistic. The corpus has been made from brochures and tourism advertising websites in the field of health tourism and sports to make a double analysis. On the one hand linguistic, by determining the type of terms, degree of specialization and existence of neologisms, on the other hand discursive, taking into account the stylistic strategies of the advertising argumentation to express the persuasion: the image and the context.
\end{abstract}

Cet article a été écrit en l'honneur de Mme la Professeure Alicja Kacprzak qui est pour moi un modèle de rigueur scientifique, de professionnalité et d'honnêteté, et dont le précieux conseil m'accompagne toujours dans la vie professionnelle et personnelle.

\section{INTRODUCTION}

Dans ce travail nous nous proposons de décrire l'aspect lexical et discursif des messages publicitaires promouvant le tourisme de santé qui s'est développé d'une façon extraordinaire dans notre société et qui est en pleine croissance grâce aux agences de voyages et aux tour-opérateurs spécialisés dans ce secteur.

Quand il est question de décrire le vocabulaire propre à un champ lexical et à un type de discours, il est nécessaire de cerner leur aspect le plus caractéristique. 
L'objet de notre étude sera d'analyser le lexique du tourisme de santé et des sports dans les textes de promotion touristique et les aspects discursifs de leur finalité persuasive.

Dans un premier temps, nous nous posons la question de la spécificité de ce type de discours : où faut-il la chercher? Est-ce dans les termes, les contextes ou les objectifs du discours publicitaire ? Il nous semble qu'elle réside plutôt dans le discours, dans la finalité persuasive du message publicitaire, aussi l'approche purement lexicale peut-elle paraitre réductionniste. Il faut aller plus loin et entrer dans le domaine de la sémiotique et de l'extralinguistique.

Le corpus sur lequel est fondée cette étude provient de brochures et de sites web promouvant le tourisme de santé et le sport. Nous avons envisagé de le soumettre à une double analyse : linguistique d'une part, consistant à examiner le type des termes, leur degré de spécialisation et le recours aux néologismes, et discursive d'autre part, tenant compte des stratégies stylistiques de l'argumentation publicitaire ayant pour but la persuasion à travers l'image et le contexte.

Une réflexion théorique sur la dénotation et la connotation précédera notre analyse.

\section{Mots dénotatifs ou Évocateurs?}

En suivant Romero Gualda (2005b : 184), on peut affirmer a priori que, loin d'être réservée aux spécialistes, la langue de la publicité est censée influencer un destinataire non professionnel - un public parfois très précis et ciblé, mais général. Autrement dit, le langage médical ou sportif, employés dans la publicité touristique et dans les médias, arrivent banalisés aux récepteurs du message et sont tout à fait compréhensibles pour les usagers ordinaires de la langue. Cette banalisation, dont R. Galisson parle déjà en 1978 dans Recherches de lexicologie descriptive : la banalisation lexicale, peut concerner n'importe quelle langue, soit-elle technique, professionnelle ou sectorielle; elle survient quand les termes sont connus par les sujets parlants qui ne partagent pas la même activité professionnelle.

Dans ce contexte, dénotation, connotation et identification de mots évocateurs, sans négliger leur fonction référentielle, feront l'objet de notre étude. Notre analyse linguistique consistera dans l'observation du type d'unités lexicales utilisées. Nous allons aussi déterminer les catégories grammaticales et les constructions syntaxiques prédominantes dans les discours publicitaires.

Néanmoins, quoique la fonction poétique du langage publicitaire soit très importante, il ne faut pas négliger la fonction référentielle des mots et des termes. Les signes linguistiques doivent activer différentes fonctions du langage et, pour 
l'analyser, il faut toujours tenir compte du texte et du contexte. Ainsi par exemple, dans le texte publicitaire ${ }^{1}$ Besoin de se faire chouchouter, faire le plein d'oligoéléments?, le terme oligo-éléments, qui relève de la chimie, joue un rôle référentiel. Cependant, si on analyse le reste du texte : Choisissez la remise en forme Privilège avec un modelage tous les jours, et la photo suivante d'un bel homme relaxé en plein massage, on remarque que, dans ce cas, outre la fonction référentielle, c'est la fonction évocatrice de la qualité de vie et de la santé que le récepteur active dans le contexte d'une annonce publicitaire, ce pour quoi les connaissances en chimie ne sont pas nécessaires pour la compréhension du message.

Ceci étant, il est essentiel de rappeler les deux aspects d'un terme : dénotatif (le sens) et connotatif (l'évocation). La dénotation et la connotation ont le même référent, mais elles s'opposent du point de vue psychologique, l'une activant la réponse cognitive, l'autre impliquant une réponse affective ajoutée par le contexte (Romero Gualda, 2005b : 187). Dans le cas des annonces publicitaires promouvant le tourisme de santé, la connotation est d'habitude ajoutée par le biais des images, des photos évoquant le bien-être, la vitalité et la détente.

C'est justement à ces valeurs ajoutées que le publicitaire recourt pour attirer l'attention du récepteur. Voyons l'exemple des lexies océan et espace dans ce contexte. Un mot comme océan, accompagné de compléments tels que un océan de bonheur, un océan de verts, un océan de rêve, un océan de vie, un océan de saveurs, un océan de richesses ne dénote plus « une vaste étendue d'eau salée qui couvre une grande partie de la surface du globe terrestre » (Le Petit Robert), mais la côte atlantique française, attirante par ses centres de thalassothérapie (océan de bonheur), par ses espaces verts (océan de verts), par ses villes animées (océan de vie), par ses vestiges culturels (océan de richesses). Et plus encore : la lexie océan, placée à côté de belles photos, étant une métaphore de magnanimité dans ce contexte, sert à évoquer tous ces atouts, mais élevés au maximum, au degré superlatif.

Il en va de même pour le mot espace dans le slogan Espace forme : le sport plaisir. Ce lexème a ici une connotation très attirante, faisant allusion au plaisir de se sentir en forme, en pleine santé lorsqu'on pratique une activité sportive. C'est-à-dire que le sens référentiel spatial de « lieu plus ou moins délimité » ( $L e$ Petit Robert) s'est enrichi grâce aux substantifs de son co-texte, qui se qualifient mutuellement et enrichissent le sens en ajoutant de nouvelles valeurs au mot (qui échappe même à sa référence spatiale originaire) et en introduisant un aspect psychologique de bien-être et de plaisir tout à fait original.

${ }^{1} \mathrm{http}: / / \mathrm{www}$.thalassopornic.com/sejours-thalasso/cures-de-remise-en-forme/cure-remise-forme -privilege (dernière consultation : le 02.01.2018). 
D'autre part, il est intéressant d'observer aussi la classe grammaticale des mots évocateurs qui apparaissent dans ce type d'annonces, à savoir les verbes, les adjectifs et surtout les substantifs. Un substantif, offrant par nature une délimitation sémantique claire et nette, est susceptible de devenir évocateur quand il est accompagné d'un adjectif ou d'un verbe. Par exemple, dans le cas de la publicité invitant le destinataire à patiner : Redécouvrez la patinoire la plus chaleureuse à Montréal !, le mot patinoire évoque aussi une ambiance décontractée, amicale, même " jeune ", et c'est grâce à l'adjectif chaleureuse ayant a priori un sens contradictoire : techniquement, la patinoire est en glace et doit donc être gelée, pas " chaleureuse ». L'impact de cette publicité sur le récepteur peut être efficace, justement grâce à cet adjectif qui est censé attirer les amateurs du patinage.

De la même façon, dans le slogan Patinage libre à l'année et Rafting, plaisir garanti !, l'adjectif libre apporte de la flexibilité au touriste du point de vue de l'organisation de l'escapade et le syntagme plaisir garanti promet une complète satisfaction.

Dans le domaine du tourisme de santé, nous relevons également beaucoup de verbes évocateurs : Se ressourcer... Vous aimeriez! Renaître en beauté, vous en rêvez... et d'images métaphoriques très suggestives et attirantes : L'Atlantique, un héritage de châteaux de sable. Prenez une Bretagne du Sud, saupoudrez d'un nuage d'Armor, d'un parfum méditerranéen, d'un soupçon aquitain. À travers ce slogan, le récepteur est invité à profiter des bienfaits du soleil et de la mer de la Bretagne du sud, évoqués ici par le toponyme Bretagne fonctionnant comme une métonymie de l'objet pour le lieu d'origine (le soleil de la Bretagne du sud). Nuage d'Armor est l'antonomase de l'eau de toilette portant ce nom et soupçon aquitain est une métaphore des plaisirs sensoriels de la région.

\section{LES PARATERMES : GÉNÉRAUX OU SPÉCIALISÉS ?}

À côté des mots évocateurs, nous relevons dans le domaine du tourisme de santé beaucoup de mots du vocabulaire général déterminés soit par des adjectifs, soit par des noms, soit par des compléments de nom. Nous avons déjà étudié ce type de constructions dans un travail consacré à la création lexicale dans le domaine du tourisme thermal (Planelles Iváñez, 2017 : 53). Il s'agit des unités lexicales du vocabulaire général qui acquièrent un nouveau sens en combinaison avec d'autres éléments qui les déterminent. C'est ce que Estopà et Lorente (2008 : 37) appellent paratérminos. 
En principe, les paratermes appartiennent à la langue générale, mais ils deviennent spécialisés grâce à la détermination. Un bon exemple en est fourni par des unités lexicales telles que p. ex. espace, soin, bain, douche, massage, atelier, cours. Il n'y a que le lexème forfait qui provient de la langue de la gestion touristique et qui se spécialise encore plus quand il est suivi d'un adjectif ou d'un complément du nom : forfait essentiel du visage, forfait détente, forfait homme.

Espace détente, espace forme, espace minceur, espace santé et espace thalasso sont des unités lexicales spécialisées qui ajoutent au sens spatial celui des activités propres du tourisme de santé et de détente. Il en va de même pour le lexème soin : les syntagmes soin à la rose, soin aux huiles amincissantes, soin décontraction, soin zen du visage expriment des concepts spécialisés ne relevant que du domaine des traitements spécialisés de beauté.

Nous avons attesté aussi les termes atelier nutrition, atelier cuisine, atelier pâtisserie, massage relaxant, massage dynamisant, massage aux pierres chaudes, douche sous-marine, bain multi-jet, bain aromatique, qui deviennent techniques et ont pour référent des activités très spécialisées, au point que, dans la plupart des cas, il est nécessaire d'ajouter une explication pour savoir exactement en quoi consiste le traitement ou quel est son effet.

\section{LA CRÉATIVITÉ LEXICALE}

En ce qui concerne la créativité lexicale dans la publicité, on constate que la nouvelle sensibilité, dont nous avons parlé dans l'introduction et dans des travaux antérieurs (Planelles Iváñez, 2017 : 47), donne lieu à l'apparition de néologismes pour dénommer de nouveaux concepts et de nouvelles activités, et même à la naissance d'expressions indiquant de nouveaux degrés de qualité dans le domaine du tourisme de santé. Les termes choisis pour caractériser les " marques » spécifiques de ce type de tourisme, ainsi que les procédés morphosyntaxiques ou de changement de classe grammaticale dans la création lexicale, font aussi partie de la néologie dans ce domaine.

Par exemple, le terme thalassothérapie, avec ses variantes thalassolthalassa formées par troncation, sont des néologismes qui expriment non seulement une nouvelle activité mais aussi une « marque » qui appartient à un nouveau style de vie. Ainsi, on trouve Thalassothérapie en Bretagne, Formule thalasso, Thalassa, carte de la thalasso en Bretagne, Ma thalasso c'est Saint Malo.

Un autre néologisme fréquent est le sigle latin SPA (Salus Per Aquam), qui exprime soit l'endroit, soit l'ensemble d'activités et de traitements, soit le nom ou la marque de beaucoup d'établissements hôteliers offrant ce type de services. 
Ce terme a une dénotation très claire et compréhensible à l'heure actuelle pour la majorité du public. Nous pouvons même le trouver qualifié, p. ex. Spa beauté exprime le lieu où l'on suit des traitements de beauté-santé et le référent continue d'être concret et clair pour le récepteur. Il faut dire que, avec les éléments linguistiques et extralinguistiques qui accompagnent le terme en question, il évoque non seulement la santé, mais aussi le soin du corps, la recherche de la beauté éternelle et le goût pour le contact avec la nature revitalisante et soignante.

L'analyse de plusieurs sites web de thalassothérapie et de services de SPA nous a amenée à constater l'existence de beaucoup de néologismes qui font allusion aux traitements et dont le sens est a priori obscur pour un récepteur non spécialiste ou non initié au tourisme de santé-beauté. Ainsi, soins corps et soins visage désignent un ensemble de traitements, mais il est nécessaire de consulter leur contenu pour arriver à savoir en quoi consiste chacun d'entre eux et en faire le choix. Ces dénominations contiennent des éléments semi-techniques désignant soit la technique utilisée (Soin Hydratation Marine, Soin Nutrition Marine), soit le résultat obtenu (Soin Pureté Marine, Soin Douceur Marine), soit encore l'élément chimique utilisé dans le traitement (Soin Oxygénation Marine). Il faut remarquer que, dans toutes ces expressions opaques du fait de leur caractère semiscientifique, l'adjectif marine ajoute toujours la valeur curative et bienfaisante de la nature.

\subsection{LA FORMATION DES NÉOLOGISMES}

On peut affirmer que les néologismes employés dans les textes publicitaires suivent les règles générales de la formation des mots, la création de nouvelles dénominations s'effectuant le plus souvent au moyen de la composition savante : hydrojet, bain multi-jet, pressothérapie, pédiluve, algothérapie, aquagym, motoneige.

Il convient de noter que la composition populaire substantif + substantif est la plus fréquente pour former des termes dénommant de nouveaux concepts et exprimant cette nouvelle sensibilité et le goût pour les soins de la santé et de la beauté : océan fleuve, atelier nutrition, atelier cuisine, drainage visage oxygénant, cure thalasso, effet "Allier », séjours iode et sensations.

On trouve aussi des lexies complexes construites par composition à l'aide de la structure substantif + adjectif ou substantif + complément du nom, des exemples en étant fournis par des unités telles que : suivi diététique, consultation diététique, gommage exfoliant, soins à la carte, douche à pomme, mesure d'impédance, salle de fitness, cours d'abdos-fessiers. 
L'antonomase est un procédé caractéristique de la langue de la publicité pour exprimer de nouveaux sens : le nom propre, que ce soit d'une marque ou d'un site, devient un nom commun désignant un produit : l'effet "Allier »; Prenez une Bretagne du Sud, saupoudrez d'un nuage d'Armor, d'un parfum méditerranéen, d'un soupçon aquitain.

Finalement, l'emprunt est un procédé de formation de néologismes très fréquent dans le domaine des sports et des activités de loisir : fitness, cardiotraining, step, stretching, watermass.

Il ne faut pas oublier que toutes ces unités lexicales à caractère semi-technique sont conçues pour attirer le récepteur : les services et les produits promus dans les messages publicitaires sont entourés d'un halo pseudo-scientifique à but persuasif.

Les euphémismes, enfin, apparaissent aussi dans l'activité publicitaire en tant qu'éléments servant à magnifier et à dissimuler les aspects négatifs ou moins attirants des produits présentés dans la publicité touristique. Ainsi, se muscler sans peine, maigrir sans souffrir et avoir une hygiène alimentaire sont des expressions euphémistiques devenues topiques, qui continuent d'être attirantes et efficaces du point de vue de la promotion et la vente de produits de tourisme sportif et de santébeauté. Un océan fleuve est aussi, à notre avis, une expression euphémistique pour désigner la Loire comme le fleuve qui adoucit les fougues de l'Atlantique.

\section{LA SPÉCIALISATION AU-DELÀ DU MOT}

La méthodologie reste incomplète ou réductive, si l'on étudie les mots isolément. Autrement dit, notre réflexion sur les termes va au-delà du mot et se fonde aussi sur les principes de l'analyse du discours. Ainsi, nous observons dans ce corpus publicitaire la présence de divers procédés rhétoriques utilisés pour persuader le touriste et pour présenter le produit d'une façon attirante. Il s'agit d'une persuasion pragmatique et commerciale. C'est ici que les aspects extralinguistiques et sémiotiques du message publicitaire entrent en action.

Il faut s'interroger sur l'importance de l'image (cf. Vilarnovo, $2005: 43$ ) en tant qu'élément exprimant, tout comme le signe linguistique, la dénotation et la connotation dans un texte publicitaire (Romero Gualda, 2005a : 83). En outre, s'impose la question de savoir quel élément détermine le plus la signification dans le message publicitaire. Est-ce le mot ou plutôt l'image ?

Adam et Bonhomme (1997 : 177-178) présentent le modèle binaire de Roland Barthes sur l'argumentation iconique : 
Exposé principalement dans "Rhétorique et image » (1964) et ayant connu par la suite un grand succès, ce modèle s'efforce de répondre à deux interrogations complémentaires : comment la «représentation analogique » qu'est l'image publicitaire peut-elle créer de véritables systèmes de signes? ou plus précisément : comment le sens naît-il à l'image ? [...] Pour Barthes, l'image publicitaire consiste en une imbrication étroite de deux soussystèmes sémiologiques perçus simultanément, mais qu'on peut hiérarchiser.

L'image dénotée se trouve au premier niveau, tandis que l'image connotée se situe au deuxième. La première est un message sans code, et avec la deuxième on assiste à la " "venue du code" et à la genèse du sens qui se fait par l'injection de signifiés connotatifs, secondaires et culturels, sur les signifiants du niveau dénoté» (Adam, Bonhomme, ibid., p. 178).

Analysons plusieurs exemples de notre corpus. Dans la brochure de promotion institutionnelle de la région Loire-Atlantique, nous pouvons relever les slogans suivants : un océan de rêve, un océan de verts, un océan fleuve, un océan de vie, un océan de richesses, un océan de saveurs. Leur sens reste cependant obscur et même déconcertant, lorsqu'ils sont séparés des images et de leurs connotations dans les contextes. Ainsi, le syntagme isolé un océan fleuve est même une construction apparemment incorrecte du point de vue sémantique, un substantif étant modifié par un autre dont le sens est contradictoire. Au contraire, si l'on observe le contexte, l'image évoquant un fleuve immense mais calme, et que l'on lit le texte ${ }^{2}$, on comprend qu'il s'agit d'une stratégie stylistique pour montrer les contrastes naturels dont le touriste peut jouir en visitant cette région. Le sens du slogan ne se trouve pas seulement dans le texte lui-même : il doit être complété par l'image connotée et le contexte.

Est-ce le mot plus important que l'image ? Est-ce l'image plus importante que le mot? On dirait qu'ils sont complémentaires. Le texte reste incomplet sans l'image, et vice versa. Ainsi, ce n'est que l'iconotexte qui est apte à transmettre l'intention persuasive du créateur de la publicité.

${ }^{2}$ « Un océan fleuve. La Loire adoucit les fougues de l'Atlantique. L'océan vivifie le fleuve indolent. La Loire-Atlantique découle de cette double influence qui la pare de mille nuances.

D'Ancenis à l'estuaire, la Loire ouvre ses bras pour s'évanouir dans la mer. Flot de lumière [...], le grand fleuve blond déroule son paysage lascif aux rivages ondulés, voilant son passage d'un halo singulier et dévoilant une nuée d'îlots échoués où subsistent fermes et prés. Baignant dans ce doux éclairage, villes et villages suivent les caprices d'un fleuve changeant, bordé de peupliers noirs et de saules buissonnants. Il a fallu des lustres pour hisser ces feuillages froissés vers un ciel moiré, mais la Loire n'est pas pressée.

Fleuve-mémoire d'une Douce-France, royale et indifférente aux turbulences du progrès, elle traverse les manuels d'histoire et remonte le cours de l'enfance.

À Nantes, la Loire rejoint Jules Verne, délaisse ses gabares et largue les amarres ». 


\section{EN GUISE DE CONCLUSION}

Après l'analyse des textes publicitaires du tourisme de santé et du sport constituant notre corpus, nous pouvons affirmer que la spécificité de la langue ne se trouve pas nécessairement, ni exclusivement, dans le lexique. C'est plutôt dans le discours, voire dans la finalité persuasive du discours publicitaire, que nous identifions cette spécificité.

En effet, on peut constater que le lexique utilisé dans le discours publicitaire du tourisme n'est pas cryptique, c'est à dire que son rapport avec la langue commune est très généralisé. Le fait qu'il appartient à une activité si quotidienne que le tourisme, nous mène à le classer parmi les discours de vulgarisation. En plus, la nouvelle sensibilité, que l'on observe dans la société depuis quelques dizaines d'années, et le goût pour la nature, la santé, la beauté et le tourisme se rattachant à ce type d'activités, ont eu pour conséquence une autre perception de ce type de discours, considéré non plus comme un discours de spécialité médicale ou esthétique, mais comme celui relevant de la langue commune, ou plutôt, de la langue de vulgarisation où le sens des termes et des contextes est transparent.

Cette constatation a été illustrée dans notre analyse par des exemples du champ du tourisme de santé et du tourisme sportif. La conclusion est que ces « langues de spécialité » ou " sectorielles » ne provoquent pas l'occultation du sens à la communauté linguistique qui les parle, car le signifié s'explique grâce au contexte et aux images : le discours spécifique devient ainsi général.

Enfin, les images, les mots évocateurs, les métaphores, la mise en page, le contexte tout court - tous ces éléments contribuent à enrichir le sens des termes et à les rendre transparents. Ainsi, les termes tels que massage lymphatique, chromothérapie, thalassothérapie, aromathérapie, font déjà partie de notre vocabulaire commun et on peut affirmer qu'ils appartiennent à la langue de vulgarisation. En d'autres mots, la langue de spécialité devient une langue de vulgarisation grâce aux éléments extralinguistiques faisant partie du discours, ainsi qu'à la nouvelle sensibilité collective de recherche de la santé et de la beauté. En revanche, les mots de la langue commune, appelés paratermes, deviennent des termes de spécialité quand ils sont modifiés et utilisés dans le contexte particulier de la publicité : espace détente, espace forme, soin aux huiles amincissantes ou atelier cuisine en constituent des exemples convaincants.

Pour conclure, les réflexions ci-dessus montrent que le lexique n'est ni le centre ni la clé du texte publicitaire. Les éléments extralinguistiques chargés d'information et de fonctions sémiotiques constituent également une partie importante du message. La publicité de produits touristiques liés à la nouvelle sensibilité à l'égard de la santé et la beauté n'en est qu'un exemple parmi d'autres. 


\section{Références bibliographiques}

ADAM, Jean-Michel, BONHOMME, Marc (1997), L'Argumentation publicitaire. Rhétorique de l'éloge et de la persuasion, Paris, Nathan.

ESTOPÀ, Rosa, LORENTE CASAFONT, Mercè (2008), « Los paratérminos en la extracción automática de terminología ", in Actes de GLAT-BREST 2008. Terminologie. Discours, technologies et acteurs sociaux, Brest, Les presses de TELECOM Bretagne, pp. 35-47.

GALISSON, Robert (1978), Recherches de lexicologie descriptive : la banalisation lexicale, Paris, Nathan Université.

PLANELLES IVÁÑEZ, Montserrat (2017), « La création lexicale dans le domaine du tourisme thermal », in De la langue à l'expression : le parcours de l'expérience discursive. Hommage à Marina Aragón Cobo (C. Carvalho, M. Planelles Iváñez, E. Sandakova éds), Alicante, Publicaciones de la Universidad de Alicante, pp. 47-63.

ROMERO GUALDA, María Victoria (2005a), "Texto e imagen en el mensaje publicitario », in Lenguaje publicitario. La seducción permanente (M. V. Romero Gualda éd.), Barcelona, Ariel, pp. 83-87.

ROMERO GUALDA, María Victoria (2005b), " La palabra en los mensajes publicitarios », in Lenguaje publicitario. La seducción permanente (M. V. Romero Gualda éd.), Barcelona, Ariel, pp. 183-202.

SPANG, Kurt (2005), « Publicidad y retórica », in Lenguaje publicitario. La seducción permanente (M. V. Romero Gualda éd.), Barcelona, Ariel, pp. 27-42.

SPANG, Kurt (2006), Persuasión. Fundamentos de retórica, $2^{\mathrm{e}}$ éd., Pamplona, Eunsa.

VILARNOVO, Antonio (2005), «Aspectos semióticos de la publicidad», in Lenguaje publicitario. La seducción permanente (M. V. Romero Gualda éd.), Barcelona, Ariel, pp. 43-63.

\section{Sitographie}

www.thalassopornic.com/sejours-thalasso/cures-de-remise-en-forme/cure-remise-forme-privilege. 